\title{
Porcentaje de luminosidad y grano de lijas en el prendimiento del injerto del cacao en Puerto Bermúdez
}

\section{Percentage of luminosity and sandpaper bean in the firing of cocoa grafting in Puerto Bermúdez}

Mario Enrique Cayetano Llosa ${ }^{1}$, Manuel Mario Chuyma Tomaylla ${ }^{1}$, Ángel Amado Romero Cahuana ${ }^{1}$, Juana María Muñoz Espino ${ }^{1}$, Pablo Pedro Villegas Panduro ${ }^{2}$

${ }^{1}$ Universidad Nacional Intercultural de la Amazonia. Facultad de Ingeniería y Ciencias Ambientales. Ucayali, Perú. Email: mariocayetanollosa_17@hotmail.com.

${ }^{1}$ Universidad Nacional Intercultural de la Amazonia. Facultad de Ingeniería y Ciencias Ambientales. Ucayali, Perú. Email: mchuymat@unia.edu.pe. ORCID: https://orcid.org/0000-0002-7833-0267

${ }^{1}$ Universidad Nacional Intercultural de la Amazonia. Facultad de Ingeniería y Ciencias Ambientales. Ucayali, Perú. Email: aromeroc@ unia.edu.pe. ORCID: https://orcid.org/0000-0002-4790-2883

${ }^{1}$ Universidad Nacional Intercultural de la Amazonia. Facultad de Ingeniería y Ciencias Ambientales. Ucayali, Perú. Email: jmme3264@gmail.com.

${ }^{2}$ Universidad Nacional de Ucayali - Perú. Carretera Federico Basadre Km 5.5, Callería. Ucayali. Facultad de Ciencias Agropecuarias. Email: pablo_villegas@unu.edu.pe ORCID: https://orcid.org/0000-0001-9300-8113

\section{Resumen}

Se estudió la influencia de los porcentajes de luminosidad y número de granos de lijas en el prendimiento del injerto tipo púa central de Theobroma cacao L., el cual se realizó en la Comunidad Nativa San Pedro, del Distrito de Puerto Bermúdez, Provincia de Oxapampa, Departamento de Pasco. Se construyó tres viveros con un techo de malla Raschel, con porcentajes de sombra de $40 \%, 60 \%$ y $80 \%$. Las semillas para los patrones provinieron del clon CCN - 51, los cuales fueron sembradas en bolsas de 6 × 8 pulgadas conteniendo sustrato y colocadas en un vivero. Las varetas para injertar, correspondieron al clon CCN 51, las cuales fueron extraídas el mismo día del injertado. Tres navajas de injertar fueron afiladas utilizando tres tamaños de granos de lijas de agua (400-A, 600-A y 1000A) respectivamente, ejecutándose el procedimiento de injerto de púa central, para luego acondicionar los plantones injertados en los tres viveros construidos, evaluándose a los 30 y 60 días después del injertado. Se empleó el diseño Completamente al Azar (DCA) con arreglo factorial 3 x 3 , concluyéndose que, el porcentaje de luminosidad no tiene influencia para el porcentaje de prendimiento, siendo el número de grano de lija 1000-A el que logro $100 \%$ de prendimiento. Asimismo, la interacción $60 \%$ de luminosidad más el número de grano de lija 1000-A mostraron mayores promedios de longitud de brote y número de hojas, sin embargo, la interacción $80 \%$ de luminosidad más número de grano de lija 1000-A logro mayores promedios de número de brotes, y la interacción $60 \%$ de luminosidad más el número de grano de lija 400-A logró mayores promedios de diámetro de brote, finalmente, $60 \%$ y $40 \%$ de luminosidad lograron los mejores promedios de diámetro de patrón.

Palabras clave: Clon CCN-51, patrón, vareta yemera, injerto de púa central, navaja de injertar, malla Raschel. 


\begin{abstract}
The influence of the percentages of luminosity and number of sandpaper grains in the grasping of the central spike type graft of Theobroma cacao L., which was carried out in the San Pedro Native Community, of the District of Puerto Bermúdez, Province of Oxapampa, was studied. Pasco Department. Three nurseries were built with a Raschel mesh roof, with shade percentages of $40 \%$, $60 \%$ and $80 \%$. The seeds for the patterns came from the CCN-51 clone, which were sown in $6 \times 8$ inch bags containing substrate and placed in a nursery. The rods for grafting corresponded to clone CCN 51, which were extracted the same day as grafting. Three grafting blades were sharpened using three sizes of water sandpaper grains (400-A, 600-A and 1000-A) respectively, executing the central spike grafting procedure, to later condition the grafted seedlings in the three built nurseries. , evaluating at 30 and 60 days after grafting. The Completely Random Design (DCA) was used with a $3 \times 3$ factorial arrangement, concluding that the percentage of luminosity has no influence on the percentage of seizure, being the number of sandpaper grit 1000-A which achieved $100 \%$ of seizure. Likewise, the interaction $60 \%$ of luminosity plus the number of sandpaper grain 1000-A showed greater averages of shoot length and number of leaves, however, the interaction $80 \%$ of luminosity plus the number of sandpaper 1000-A achieved greater averages of number of shoots, and the interaction $60 \%$ of luminosity plus the number of sandpaper grit 400-A achieved higher averages of bud diameter, finally, $60 \%$ and $40 \%$ of luminosity achieved the best averages of pattern diameter.
\end{abstract}

Keywords: CCN-51 clone, pattern, bud bud, central barb graft, grafting knife, Raschel mesh.

\section{Introducción}

El cacao se produce en más de 50 países con una producción total mundial de 3 millones de toneladas anuales, siendo Costa de Marfil el principal productor, con el $32 \%$ del total mundial, seguido de Indonesia (14\%), Nigeria (13\%) y Ghana (10\%), siendo Ecuador y le sigue Brasil y República Dominicana, los mayores productores en Latinoamérica (MINAGRI, 2012). La importancia del cacao genera alta demanda de plantas, motivo por el cual, conocer y comprender los métodos de propagación, especialmente el injerto, es de vital importancia, ya que está influenciado por las condiciones ambientales, de la estructura morfológica y los aspectos fisiológicos y citológicos de la planta (Guevara, 2011).

Sandoya (2019) afirma que injertar consiste en tomar una yema de la rama de un árbol seleccionado y evaluado, para unirla a un patrón que es una planta generada por semilla. Esta yema una vez implantada, se activa y a medida que crece conforma la parte aérea del nuevo árbol y por lo tanto sus características genéticas son idénticas a las del árbol de donde se extrajo la yema, siendo los tipos de injertos más utilizados en cacao, el de púa central o terminal, injertación en parche, injerto de corona e injertos por aproximación. 
Gamboa (2015) estudió el comportamiento de los patrones antes del injerto y de las plantas injertadas en vivero, para el cual se utilizaron semillas de cuatro clones de cacao (IMC-67, VRAE-99, TSH-565 y UF-221), y varas yemeras de cuatro clones de cacao (VRAE99, VRAE-15, TSH-565 y CCN-51), siendo el clon IMC-67 el patrón con mayores atributos en el vivero, en tanto que el clon VRAE-15 fue el que tuvo mayores atributos como injerto en vivero, siendo los tratamientos VRAE-99/UF-221 y VRAE15/UF-221, fueron los que alcanzaron el $100 \%$ de prendimiento. Challco (2019) estudio la influencia de la edad del patrón de cacao $(120,150,180$ y 210 días) sobre el prendimiento de los injertos ICS-95 y Chuncho en "La Convención", utilizándose dos tipos de injerto el de Parche y Púa central, demostrándose que el patrón de 120 días de edad mostro el mejor porcentaje de prendimiento, con yemas del clon ICS-95 e injerto parche con el 91,67\% de prendimiento y el mejor material para injertar fue el clon ICS-95 con el 91,50 \% de prendimiento. Huarancca (2019) evaluó la influencia de las fases lunares (luna nueva, cuarto creciente, luna llena y cuarto menguante) y tipos de injertos (púa central y púa lateral) en la propagación de cacao, bajo condiciones de vivero a $620 \mathrm{msnm}$, en Pichari, Cusco, concluyendo que las fases de luna llena y cuarto menguante presentaron resultados favorables para los parámetros evaluados, días de brotación con 14.7 y 15.3 días después del injerto, porcentaje de prendimiento con $99.6 \%$ y $98.3 \%$, longitud de brotes con $13.7 \mathrm{~cm}$ y $15 \mathrm{~cm}$, número de hojas con $7.8 \mathrm{y}$ 8.8 hojas por planta, respectivamente, no existiendo diferencias estadísticas significativas para los tipos de injerto de púa central y púa lateral para los parámetros evaluados. León (2020) evaluó tres tipos de injertación en cacao (T1: púa central, T2: púa lateral y T3: parche), en los cuales el clon EET 400 se utilizó como patrón y el clon EET 103 como vareta para determinar el porcentaje de prendimiento, demostrándose que el T1 logró el mejor porcentaje de prendimiento (88\%), seguido del T2 (71\%) siendo el T3 el que mostro un bajo porcentaje de prendimiento (13\%).

La investigación evaluó la influencia de los porcentajes de luminosidad y granos de lijas en el prendimiento del injerto tipo púa central de Theobroma cacao L. bajo condiciones de Puerto Bermúdez. 


\section{Métodos}

La investigación se realizó en el fundo Vista Hermosa, ubicado en la Comunidad Nativa San Pedro, del Distrito de Puerto Bermúdez, Provincia de Oxapampa, Departamento de Pasco, en la coordenada UTM 18L 506346 8860871 y una altitud de $257 \mathrm{msnm}$. La temperatura media anual es de $25,4{ }^{\circ} \mathrm{C}$, con temperatura máxima $36,5^{\circ} \mathrm{C}$, y mínima de $17,4{ }^{\circ} \mathrm{C}$, la humedad relativa es de $84,5 \%$, siendo la precipitación media anual, de 1773,44 mm/año (Baldoceda 1993).

Se construyó tres viveros de $2 \mathrm{~m}$ de ancho y 4 $\mathrm{m}$ de largo, con un techo de malla raschell, con un porcentaje de sombra de $40 \%, 60 \%$ y $80 \%$.

Las semillas provinieron de plantas madres del clon CCN - 51 (se tomaron solo las semillas de la parte central, el resto se descartó), a las cuales se les eliminó el mucílago frotándolo con aserrín de bolaina, para luego ser desinfectados con Oxicloruro de cobre 50\%, para proceder a sembrarlas en bolsas de alta densidad 6 x 8 pulgadas conteniendo un sustrato constituido por tierra negra, materia orgánica y arena de rio previamente zarandeadas en una malla de $1 \mathrm{~cm} 2$, mezcladas y desinfectados con agua hirviente. Las bolsas sembradas fueron colocadas en un vivero bajo sombra parcial (70 - 89\% de luminosidad), en los cuales, las semillas comenzaron a germinar.

Las varetas portadoras de yemas para injertar, provinieron de una parcela de cinco años de producción, correspondiente al clon CCN 51, ubicado en las coordenadas UTM 18L 506346 8860871 y una altitud de $257 \mathrm{msnm}$, correspondiente al Distrito de Puerto Bermúdez, Provincia de Oxapampa, Departamento de Pasco, las cuales fueron extraídas el mismo día en el que se ejecutó el injerto. Las varetas presentaron un grosor similar al patrón $(7 \mathrm{~mm})$, descartando los extremos más verdoso o tierno y más viejo u oscuro, cuidando que contenga 3 a 4 yemas vegetativas ligeramente brotadas, cortándole las hojas y dejando una parte del pecíolo.

Para el afilado de las tres navajas de injertar marca Bahco P11, modelo recto, se utilizó tres tamaños de granos de lijas de agua (400-A, 600-A y 1000-A) para cada navaja respectivamente, ejecutándose el procedimiento de injerto de púa central (Somarriba, et al. 2010), desvendándose a los 30 días de haberse realizado el injertado. Los plantones injertados fueron acondicionados en los tres viveros construidos con techo de malla raschell, con $40 \%, 60 \%$ y $80 \%$ de luminosidad 
respectivamente, para ser evaluados a los $30 \mathrm{y}$

60 días después del injertado.

Se empleó el diseño Completamente al Azar (DCA) con arreglo factorial $3 \times 3$, siendo el factor $\mathrm{A}=$ porcentaje de luminosidad $(40 \%$, $60 \%$ y $80 \%$ ), y el factor $\mathrm{B}=$ número de grano de lija (400-A, 600-A y 1000-A), haciendo un total de 9 combinaciones, con 27 repeticiones y un total de 243 plantones. Los resultados fueron analizados empleando el software STATGRAPHICS Centurión XV.

\section{Resultados y discusión}

\section{Porcentaje de prendimiento}

La tabla 1 muestra la comparación múltiple de Tukey para el porcentaje de prendimiento en el injerto de cacao tipo púa central, observándose diferencia significativa para los porcentajes de prendimiento en cuanto al número de grano de lija 1000-A, presentando una media: $100.00 \pm 2.91$, ya que al poseer $\operatorname{los}$ granos más finos eleva el porcentaje de prendimiento ya que la cicatrización entre el injerto y patrón es más uniforme y la trasferencia de savia a través del xilema y floema es más fluida; en cuanto porcentaje de luminosidad no existe diferencia significativa sin embargo existe diferencia porcentual en el prendimiento con una media $98.67 \pm 2.91$ para el tratamiento $60 \%$ de luminosidad, debido a diversos factores como la compatibilidad del injerto y el patrón, la técnica del injertador, las condiciones ambientales y la época del injerto, como lo señala ICT (2004), quien recomienda el uso del injerto tipo púa central o lateral, por su facilidad de ejecución, disponibilidad de material vegetativo y por haber demostrado mayor prendimiento en campo con los agricultores. Asimismo, García (1988) en sus investigaciones realizadas en Tingo María (641 msnm) comparó los métodos de injerto U invertida y escudete, obteniendo prendimientos de $63.79 \%$ y $42.90 \%$, respectivamente, al igual que More (2003), quien utilizó el injerto tipo escudete y logró prendimientos entre 60 y 85 por ciento en un lapso de 60 días después del injerto.

\section{Tabla 1}

Porcentaje de prendimiento.

\begin{tabular}{cccc}
\hline Porcentaje de luminosidad & Cantidad & Prendimiento & Grupos homogéneos \\
\hline $60 \%$ & 81 & $98.67 \pm 2.91$ & $\mathrm{a}$ \\
$80 \%$ & 81 & $92.33 \pm 2.91$ & $\mathrm{a}$
\end{tabular}


$40 \%$

Número de grano de lija

1000-A

600-A

$400-\mathrm{A}$
$81 \quad 91.33 \pm 2.91$

$81 \quad 100.00 \pm 2.91$

81

$97.33 \pm 2.91$

81

$85.00 \pm 2.91$ a

a

$\mathrm{ab}$

b

${ }^{1}$ Los datos corresponden a la media \pm SD, para $n=81$.

Letras de superíndices diferentes indican diferencia significativa.

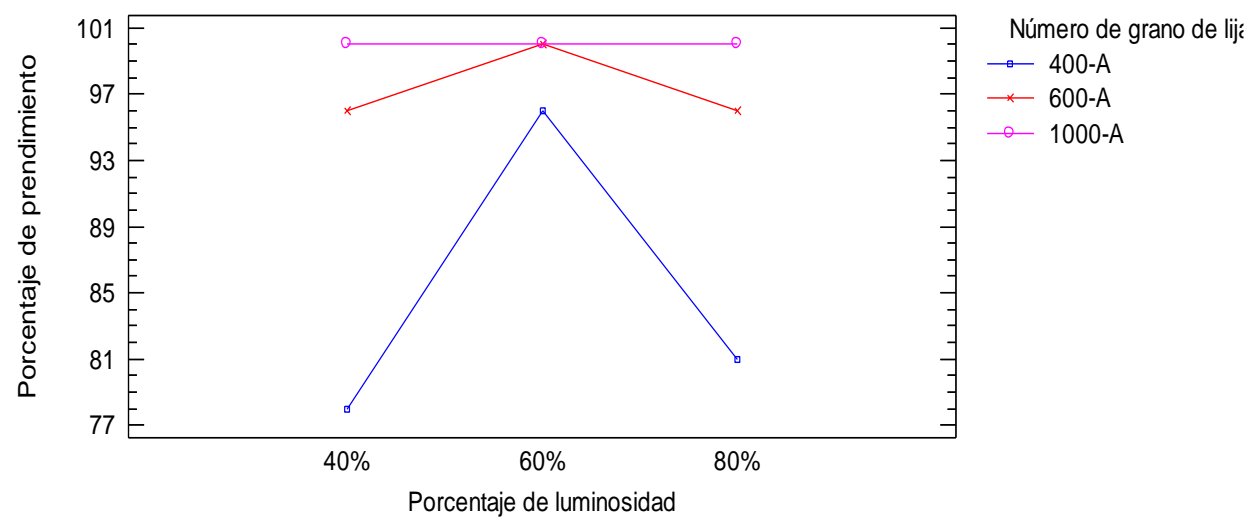

Figura 1: Interacción de los factores porcentaje de prendimiento y luminosidad en el porcentaje de prendimiento.

La figura 1 muestra la interacción entre el porcentaje de luminosidad y número de grano de lija, observándose que, conforme se incrementa los porcentajes de luminosidad en relación con el número de granos de lija 1000A presenta un porcentaje de prendimiento similar, sin embargo, conforme se incrementa los porcentajes de luminosidad del $60 \%$ hasta el $80 \%$ en relación con el grano de lija 600-A y 400-A respectivamente el porcentaje de prendimiento disminuye, demostrándose que se debe utilizar el número de grano de lija
1000-A en el afilado de la navaja injertera con una variante de luminosidad del $60 \%$, lo cual generará mejor prendimiento en el injerto de cacao tipo púa central, debido a la afinidad del corte, que conlleva a la unión más liza entre el corte patrón-púa, así mismo esto garantiza el prendimiento del injerto, lo cual es corroborado por Loria (2005) quien afirma que al realizar el injerto de púa central, las posibilidades de que el cambium del patrón entre en contacto con el cambium del injerto son mayores. 


\section{Número de brotes}

La tabla 2 muestra la comparación múltiple de Tukey para el números de brotes en el injerto tipo púa central a los 30 y 60 días después del injerto, observándose que no existen diferencias significativas para el factor porcentaje de luminosidad a los 30 y 60 días de injertado, observándose diferencias significativas para el factor número de grano de lija, en el cual, los granos de lija de 1000-A y 600-A presentaron mayor número de brotes a los 30 y 60 días de injertado, superando al grano de lija 400-A. Al respecto, Paredes (2010) obtuvo 4.40 brotes/planta con el injerto púa central y 4.20 brotes/planta con el de empalme 100 días después del injerto, y Quiroz (2005) uso bolsa plástica para cubrir el injerto, el cual influenció en la capacidad de brotamiento de la planta injertada, evitando la deshidratación del injerto y generando un microclima estable que favorece la velocidad de brotación.

Tabla 2

Numero de brotes

\begin{tabular}{cccc}
\hline Porcentaje de luminosidad & Cantidad & 30 días & 60 días \\
\hline $80 \%$ & 81 & $2.67 \pm 0.13 \mathrm{a}$ & $3.79 \pm 0.13 \mathrm{a}$ \\
$60 \%$ & 81 & $2.43 \pm 0.13 \mathrm{a}$ & $3.43 \pm 0.13 \mathrm{a}$ \\
$40 \%$ & 81 & $2.41 \pm 0.13 \mathrm{a}$ & $3.43 \pm 0.13 \mathrm{a}$ \\
$\begin{array}{cccc}\text { Número de grano de lija } \\
\text { 1000-A }\end{array}$ & & & \\
600-A & 81 & $3.44 \pm 0.13 \mathrm{a}$ & $4.60 \pm 0.13 \mathrm{a}$ \\
400-A & 81 & $2.14 \pm 0.13 \mathrm{a}$ & $3.16 \pm 0.13 \mathrm{a}$ \\
& 81 & $1.93 \pm 0.13 \mathrm{~b}$ & $2.93 \pm 0.13 \mathrm{~b}$ \\
\hline $\begin{array}{l}{ }^{1} \text { Los datos corresponden a la media } \pm \text { SD, para } \mathrm{n}=81 \\
\text { Letras de superíndices diferentes indican diferencia significativa. }\end{array}$
\end{tabular}

La figura 2 muestran el comportamiento de la interacción de los factores porcentaje de luminosidad y el número de lija en el números de brotes en el injerto de cacao tipo púa central a $\operatorname{los} 30$ y 60 días después del injerto, observándose que un $80 \%$ de lumnosidad mas el número de lija 1000-A generaron mayor número de brotes, mostrando resultados satisfactorios para el numero de brotes, debido a que la cicatrización entre el injerto y patrón es más uniforme y la trasferencia de savia a través del xilema y floema es más fluida 
UNIVERSIDAD NACIONAL DE UCAYALI

Revista de Investigación Universitaria

Versión electrónica 2664 - 8423

ARTICULO ORIGINAL
Vol. $11 \mathrm{~N}^{\circ} 2$, pp. 677 - 692, julio/diciembre 2021

Recibido 23/09/2021

Aceptado 18/12/2021

Publicado 30/12/2021

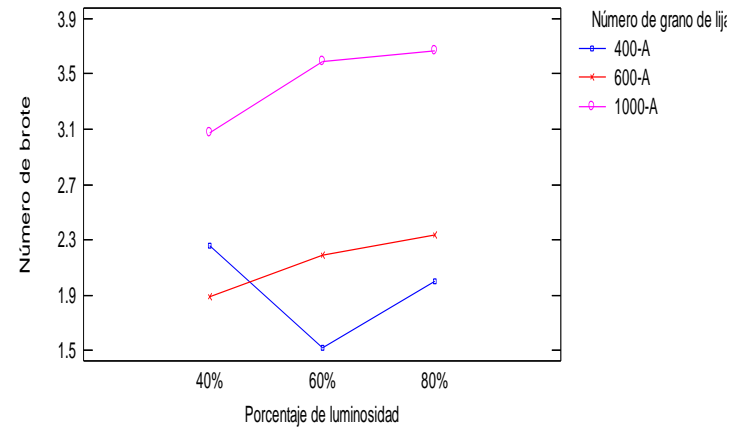

30 días después del injertado

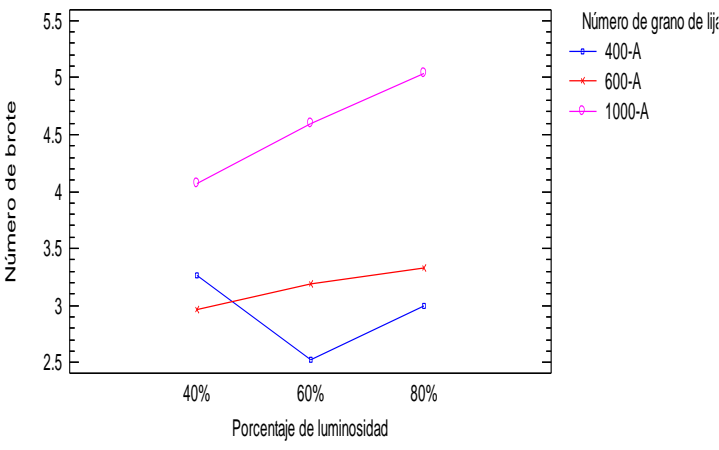

60 días después del injertado

Figura 2: Interacción de los factores porcentaje de prendimiento y luminosidad en el número de brotes.

\section{Longitud del brote}

La tabla 3 muestra la comparación múltiple de Tukey para la longitud del brote en el injerto de cacao tipo púa central a los 30 y 60 días después del injerto, observándose diferencia estadísticamente significativa en cuanto al porcentaje de luminosidad, en el cual, el $60 \%$ presentó el mejor promedio de longitud de brote a los 60 días después del injertado, y el número de lija 400-A mostró el mejor promedio de longitud de brote a los 30 y 60 días después del injertado, siendo estos resultados superiores a los reportados por Jara (1991), el cual utilizó el injerto tipo parche, con una longitud de brote entre 7.12 y $9.80 \mathrm{~cm}$, a los 90 días después del injerto. La deficiencia de luz en los brotes influye positivamente en el alargamiento puesto que aparentemente en un $60 \%$ sea el adecuado para un rápido alargamiento.

Tabla 3

Longitud del brote $(\mathrm{cm})$

\begin{tabular}{|c|c|c|c|}
\hline Porcentaje de luminosidad & Cantidad & 30 días & 60 días \\
\hline $60 \%$ & 81 & $9.10 \pm 0.26 \mathrm{a}$ & $11.62 \pm 0.26 \mathrm{a}$ \\
\hline $80 \%$ & 81 & $8.58 \pm 0.26 \mathrm{a}$ & $10.69 \pm 0.26 b$ \\
\hline $40 \%$ & 81 & $7.84 \pm 0.26 b$ & $9.72 \pm 0.26 c$ \\
\hline \multicolumn{4}{|l|}{ Número de grano de lija } \\
\hline $400-\mathrm{A}$ & 81 & $9.35 \pm 0.26 \mathrm{a}$ & $10.09 \pm 0.26 \mathrm{a}$ \\
\hline
\end{tabular}




$\begin{array}{cccc}600-\mathrm{A} & 81 & 8.27 \pm 0.26 \mathrm{a} & 11.11 \pm 0.26 \mathrm{~b} \\ 1000-\mathrm{A} & 81 & 7.91 \pm 0.26 \mathrm{~b} & 10.83 \pm 0.26 \mathrm{ab}\end{array}$

${ }^{1}$ Los datos corresponden a la media \pm SD, para $n=81$

Letras de superíndices diferentes indican diferencia significativa.

La figura 3 muestran el comportamiento de la interacción de los factores porcentaje de luminosidad y número de grano de lija para la longitud del brote en el injerto del cacao,

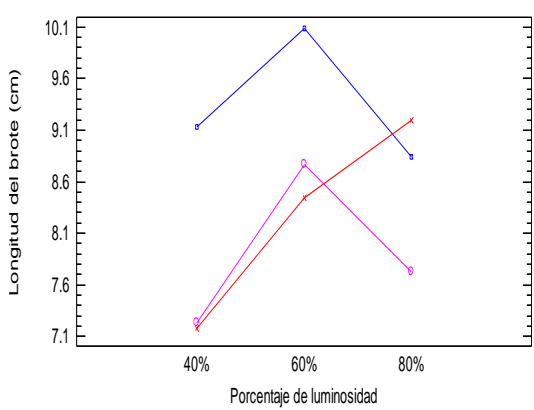

30 días después del injertado siendo el número de granos de lijas 400-A y 1000-A más un $60 \%$ de luminosidad, en la cual se registran la mayor longitud de brote a los 30 y 60 días después del injertado.

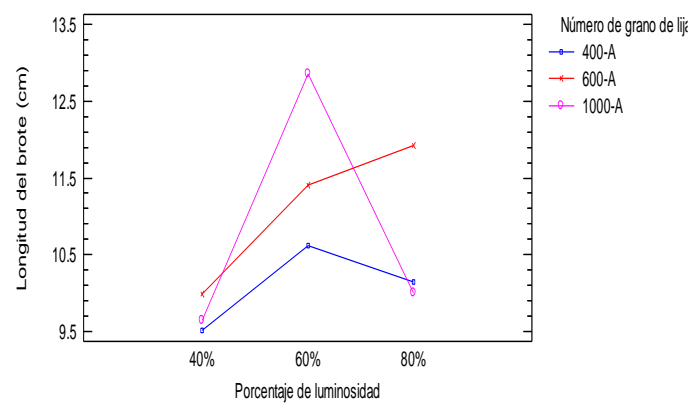

60 días después del injertado

Figura 3: Interacción de los factores porcentaje de prendimiento y luminosidad en la longitud del brote.

\section{Diámetro de brote (mm)}

La tabla 4 muestra la comparación múltiple de Tukey para el diámetro de brote del injerto de cacao tipo púa central a los 30 y 60 días después del injerto, observándose que existe diferencias estadísticamente significativas para el porcentaje de luminosidad, siendo el $60 \%$ y $80 \%$ de luminosidad los que mostraron los mayores promedios de diámetro de brote, asimismo, no se observaron diferencias significativas para el número de grano de lija a los 30 y 60 días después del injertado. 
Tabla 4

Diámetro de brote $(\mathrm{mm})$

\begin{tabular}{|c|c|c|c|}
\hline Porcentaje de luminosidad & Cantidad & 30 días & 60 días \\
\hline $60 \%$ & 81 & $1.94 \pm 0.05 \mathrm{a}$ & $3.44 \pm 0.05 \mathrm{a}$ \\
\hline $80 \%$ & 81 & $1.93 \pm 0.05 \mathrm{a}$ & $3.43 \pm 0.05 \mathrm{a}$ \\
\hline $40 \%$ & 81 & $1.63 \pm 0.05 b$ & $3.13 \pm 0.05 b$ \\
\hline \multicolumn{4}{|l|}{ Número de grano de lija } \\
\hline $1000-\mathrm{A}$ & 81 & $1.88 \pm 0.05 \mathrm{a}$ & $3.38 \pm 0.05 \mathrm{a}$ \\
\hline $600-A$ & 81 & $1.83 \pm 0.05 \mathrm{a}$ & $3.33 \pm 0.05 \mathrm{a}$ \\
\hline 400-A & 81 & $1.80 \pm 0.05 \mathrm{a}$ & $3.30 \pm 0.05 \mathrm{a}$ \\
\hline
\end{tabular}

${ }^{1}$ Los datos corresponden a la media \pm SD, para $n=81$

Letras de superíndices diferentes indican diferencia significativa.

La figura 4 muestran el comportamiento del diámetro del brote en el injerto de cacao tipo púa central según el porcentaje de luminosidad y número de grano de lija a los 30 y 60 días después injertado.

En los gráficos de interacción se puede apreciar que la luz 60 por ciento, influye positivamente en el diámetro del brote, con el grano de lija 400-A; mientras que para los otros tratamientos muestran valores inferiores en cuento al diámetro del brote del injerto, estos valores son inferiores de acuerdo a lo encontrado por Gamboa (2015) en el cual el injerto de cacao presentó un desarrollo de 3.43 $\mathrm{mm} /$ brote, así mismo superiores a lo reportado por Jara (1991) que utilizando un injerto tipo parche, encontró valores entre 2.60 y $3.00 \mathrm{~mm}$. Por otro lado, More (2003) manifiesta que el crecimiento del injerto en longitud y diámetro depende de su constitución genética y el medio ambiente. Así mismo, estos tratamientos presentan un efecto positivo en cuanto al desarrollo fisiológico del injerto. 
UNIVERSIDAD NACIONAL DE UCAYALI

Revista de Investigación Universitaria

Versión electrónica 2664 - 8423

ARTICULO ORIGINAL
Vol. $11 \mathrm{~N}^{\circ}$ 2, pp. 677 - 692, julio/diciembre 2021

Recibido 23/09/2021

Aceptado 18/12/2021

Publicado 30/12/2021

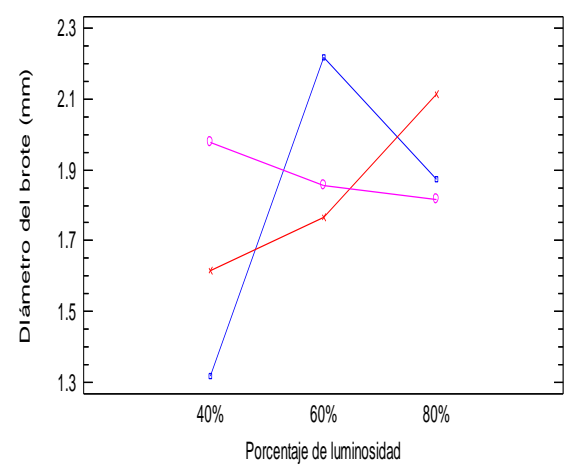

30 días después del injertado

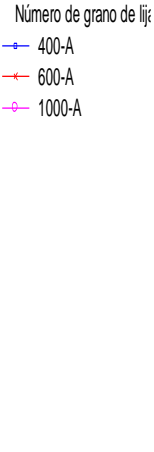

$-400-A$

* 600-A

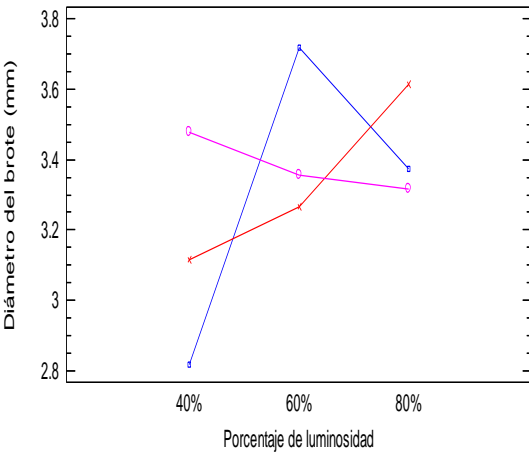

60 días después del injertado.
Número de grano de lï:

$\rightarrow$ 400-A

* 600-A

$\rightarrow$ 1000-A

Figura 4: Interacción de los factores porcentaje de prendimiento y luminosidad en el diámetro de brote.

\section{Número de hojas de brotes}

La tabla 5 muestra la comparación múltiple de Tukey para el número de hojas de brote del injerto de cacao tipo púa central a los 30 y 60 días después del injerto, observándose diferencias estadísticamente significativas para $80 \%$ y $40 \%$ de luminosidad, los cuales mostraron los mayores promedios de número de hojas, asimismo, el número de grano de lija 1000-A mostró el mayor promedio de numero de hojas a los 30 y 60 días después del injertado.

Tabla 5

Numero de hojas

\begin{tabular}{cccc}
\hline Porcentaje de luminosidad & Cantidad & 30 días & 60 días \\
\hline $80 \%$ & 81 & $4.08 \pm 0.15 \mathrm{a}$ & $5.08 \pm 0.15 \mathrm{a}$ \\
$40 \%$ & 81 & $3.96 \pm 0.15 \mathrm{a}$ & $4.96 \pm 0.15 \mathrm{a}$ \\
$60 \%$ & 81 & $3.14 \pm 0.15 \mathrm{~b}$ & $5.79 \pm 0.15 \mathrm{~b}$
\end{tabular}

Número de grano de lija

$\begin{array}{llll}1000-\mathrm{A} & 81 & 4.49 \pm 0.15 \mathrm{a} & 6.16 \pm 0.15 \mathrm{a} \\ 400-\mathrm{A} & 81 & 3.43 \pm 0.15 \mathrm{~b} & 4.77 \pm 0.15 \mathrm{~b} \\ 600-\mathrm{A} & 81 & 3.26 \pm 0.15 \mathrm{~b} & 4.91 \pm 0.15 \mathrm{~b}\end{array}$

${ }^{1}$ Los datos corresponden a la media \pm SD, para $n=81$

Letras de superíndices diferentes indican diferencia significativa. 
La figura 5 muestran el comportamiento de la interacción entre los factores porcentaje de luminosidad y número de grano de lija a los 30 y 60 días después del injerto, observándose que a los 30 días después del injertado, $80 \%$ de luminosidad más el número de grano de lija 1000-A mostraron el mejor promedio de número de hojas, y a los 60 días después del injertado, $60 \%$ de luminosidad más el número de grano de lija 1000-A mostraron el mejor promedio de número de hojas, siendo estos

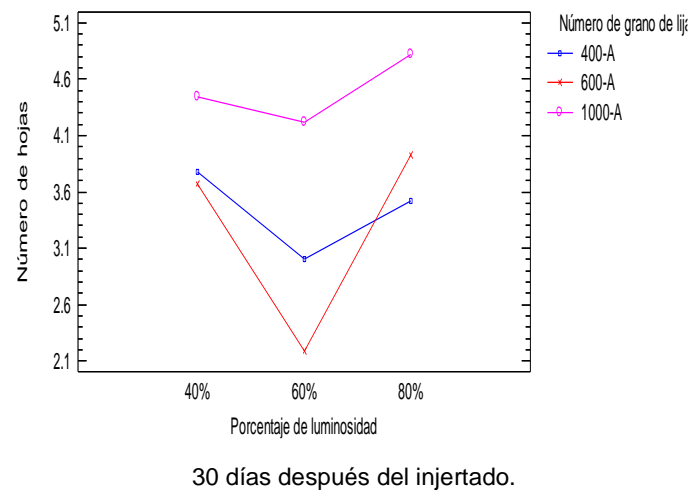

resultados superiores a lo demostrado por Jara (1991) en un ensayo a una altura 660 msnm y trabajando con el injerto tipo parche, en el cual reportó entre 4.80 y 5.65 hojas en el brote mayor y menor respectivamente, sin embargo, Vera y López (2007) reportaron que el injerto de púa central es el más recomendable para especies forestales, ya que favorece el mayor número de hojas, teniéndose en cuenta que el cacao en su primera etapa de desarrollo, no requiere de una luminosidad al 100\%.

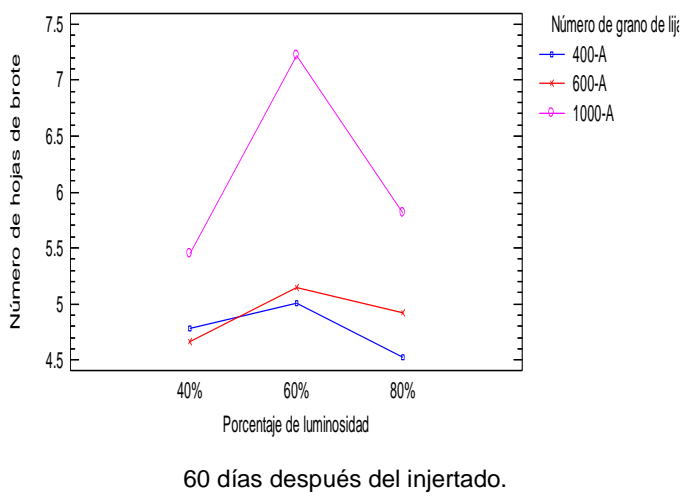

Figura 5: Interacción de los factores porcentaje de prendimiento y luminosidad en el número de hojas.

\section{Diámetro del patrón}

La tabla 6 muestra la comparación múltiple de Tukey para el diámetro del patrón en el injerto de cacao tipo púa central a los 30 y 60 días después del injerto, observándose diferencias estadísticamente significativas el porcentaje de luminosidad, en el cual, el $60 \%$ y $40 \%$ de luminosidad mostraron los mejores promedios de diámetro del patrón a los 30 y 60 días después del injertado, mostrándose diferencias significativas a los 30 días para el numero de grano de lija, siendo el número de grano de lija 1000-A el que mostró el mejor promedio de diámetro de patrón, no mostrándose 
diferencias significativas entre los números de granos de lijas a los 60 días después del injertados. Al respecto, More (2003) estudio procesos de injerto de cacao reportaron valores entre 9.93 y $11.16 \mathrm{~mm}$ de diámetro, asimismo, Chaycoj (2005), señala un diámetro de $10 \mathrm{~mm}$ para patrones a los 90 días de edad. Sin embargo, estos resultados son superiores a los encontrados por Vera y Moran (2012), los que reportaron valores entre 7.90 y $8.10 \mathrm{~mm}$, para patrones con 150 días de edad. También Carhuaz (2001), encontró que el diámetro del patrón varió de 6.5 a 10.6, en plantas de 180 días de edad. Por lo tanto, podemos afirmar que para obtener un diámetro especifico en el patrón solo se requiere de 45 a 60 días para obtener resultados óptimos en las plantaciones de cacao.

\section{Tabla 6}

Diámetro del patrón ( $\mathrm{mm})$

\begin{tabular}{|c|c|c|c|}
\hline Porcentaje de luminosidad & Cantidad & 30 días & 60 días \\
\hline $60 \%$ & 81 & $8.53 \pm 0.09 \mathrm{a}$ & $9.48 \pm 0.07 \mathrm{a}$ \\
\hline $40 \%$ & 81 & $8.29 \pm 0.09 a$ & $9.55 \pm 0.07 \mathrm{a}$ \\
\hline $80 \%$ & 81 & $8.21 \pm 0.09 \mathrm{a}$ & $9.22 \pm 0.07 b$ \\
\hline \multicolumn{4}{|l|}{ Número de grano de lija } \\
\hline $1000-\mathrm{A}$ & 81 & $8.52 \pm 0.09 \mathrm{a}$ & $9.45 \pm 0.07 \mathrm{a}$ \\
\hline $600-A$ & 81 & $8.37 \pm 0.09 \mathrm{ab}$ & $9.42 \pm 0.07 \mathrm{a}$ \\
\hline $400-A$ & 81 & $8.15 \pm 0.09 b$ & $9.38 \pm 0.07 \mathrm{a}$ \\
\hline
\end{tabular}

${ }^{1}$ Los datos corresponden a la media \pm SD, para $n=81$

Letras de superíndices diferentes indican diferencia significativa.

\section{Conclusión}

El porcentaje de luminosidad no tiene influencia para el porcentaje de prendimiento, siendo el número de grano de lija 1000-A el que logro valores de $100 \%$ de prendimiento.

La interacción $60 \%$ de luminosidad más número de grano de lija 1000-A mostraron mayores promedios de longitud de brote y número de hojas, sin embargo, la interacción $80 \%$ de luminosidad más número de grano de lija 1000-A logró mayores promedios de número de brotes, y la interacción $60 \%$ de luminosidad más el número de grano de lija 400-A logró mayores promedios de diámetro de brote, finalmente, $60 \%$ y $40 \%$ de 
luminosidad lograron los mejores promedios de diámetro de patrón.

\section{Referencia bibliográfica}

Baldoceda, R. (1993). Diagramas bioclimáticos de la zona de Pucallpa y Atalaya. Universidad Nacional de Ucayali, Pucallpa, Perú. 21p.

Carhuaz, M. (2001). Desarrollo del fruto y momento óptimo de la semilla de cacao híbrido forastero (Theobroma cacao L.) en el valle de Satipo. Tesis Magister Scientiae. Universidad Nacional Agraria La Molina. 108p.

Challco Chariarse, O. R. (2019). Influencia de la edad del patrón de cacao (Theobroma cacao L.) sobre el prendimiento de los injertos ICS-95 Y Chuncho en el sector de Macamango Distrito de Santa Ana - La Convención. Tesis para optar el título profesional de Ingeniero Agrónomo Tropical. Universidad Nacional de San Antonio Abad del Cusco. Facultad de Ciencias Agrarias. Escuela Profesional de Agronomía Tropical. Quillabamba, La Convención, Cusco. Disponible en: http://repositorio.unsaac.edu.pe/bitstr eam/handle/20.500.12918/4442/253T
20190475_TC.pdf?sequence $=1 \&$ isAl

lowed=y

Chaycoj, L. (2005). Evaluación del prendimiento de injerto de cacao (Theobroma cacao L.) UF- 667 en cinco etapas de crecimiento del patrón POUND-7. Tesis Ing Agro Universidad de San Carlos de Guatemala. 28-37p.

Gamboa Auqui, R. J. (2015). Comportamiento en vivero de cuatro clones de cacao (Theobroma cacao L.) sobre diferentes patrones en Satipo. Tesis para optar el título de Ingeniero Agrónomo. Universidad Nacional Agraria La Molina. Facultad De Agronomía Lima-Perú. Disponible en: https://repositorio.lamolina.edu.pe/bit stream/handle/UNALM/949/T007354 .pdf? sequence $=1 \&$ is Allowed $=y$

Guevara, M. (2011). Efecto de dos tipos de injerto de hendidura con tres tipos de vara yemera y con dos formas de protección en cacao (Theobroma cacao L.) en Santa Lucia- Aucayacu Tesis, Universidad Nacional Agraria De La Selva, Tingo María, Perú.

García, G. (1988). Evaluación de dos métodos de injerto en diferentes edades de patrón y con tratamiento a la vara 
yemera en cacao (Theobroma cacao

L.) en Tingo María. Tesis Ing. Agro.

Universidad Nacional Agraria de la Selva Tingo María. 45p.

Huarancca Aspur, J. C. (2019). Fases lunares y tipos de injertos en la propagación de cacao (Theobroma cacao L.), Vivero Pichari Alta 620 Msnm, Cusco, 2018. Tesis para obtener el título profesional de Ingeniero Agroforestal. Universidad Nacional de San Cristóbal de Huamanga. Facultad de Ciencias Agrarias. Escuela Profesional de Ingeniería Agroforestal. Ayacucho, Perú. Disponible en: http://repositorio.unsch.edu.pe/bitstre am/handle/UNSCH/3601/TESIS\%20 AF10_Hua.pdf?sequence=1\&isAllow $\mathrm{ed}=\mathrm{y}$

ICT. (2004). Cacao. manejo integrado del cultivo y transferencia de tecnología en la Amazonía Peruana. 33-39p.

Jara, M. (1991). Efecto del corte del patrón sobre el crecimiento y desarrollo inicial del injerto del cacao (Theobroma cacao L) en Tingo María. Tesis Ing. Agro. Universidad Nacional Agraria de la Selva. Tingo María. 51p.
León Torres, C. M. (2020). Evaluación de tres métodos de injertación en cacao (Theobroma cacao) en el cantón Jipijapa provincia de Manabí. Trabajo para obtener el título de Ingeniero Agropecuario. Universidad Estatal del Sur de Manabí. Facultad de Ciencias Naturales y de la Agricultura. Disponible en: http://repositorio.unesum.edu.ec/bitstr eam/53000/2548/1/LE\%C3\%93N\%2 0TORRES\%20TESIS\%20FINALIZ ADA.pdf

Loría, C. (2005). El injerto: Alternativa de propagación vegetativa en el cultivo de la uva (Vitis vinífera) en Costa Rica. Costa Rica. Rev. Agr. Trop. 35: 101-106 p.

MINAGRI. (2012). El cultivo del cacao en la Amazonía Peruana. Perú. 19p.

More, M. (2003). Inducción e injertación de brotes orto trópicos con fines de renovación en el cacaotero (Theobroma cacao L.) en Tingo María. Tesis Ing. Agro. Universidad Nacional Agraria de la Selva Tingo María.98p.

Paredes, R. O. (2010). Propagación vegetativa por injerto de bolaina blanca (Guazuma crinita Mart) bajo 
condiciones controladas en Pucallpa,

Perú. Tingo María, Perú.

Quiroz, C. (2005). El injerto: alternativa de propagación vegetativa el cultivo de la uva (Vitis vinífera L.). información técnica. Rev. Agro. No 35 Costa Rica. $6 \mathrm{p}$.

Sandoya Jiménez, M. J. (2019). Tipos de injertos en plantas de vivero de cacao nacional (Theobroma cacao L.). Trabajo para obtener el título de Ingeniero Agrónomo. Universidad Técnica de Babahoyo. Facultad de Ciencias Agropecuarias. Carrera de Ingeniería Agronómica. Babahoyo, Los Ríos, Ecuador. Disponible en: http://dspace.utb.edu.ec/bitstream/han dle/49000/6813/E-UTB-FACIAGING\%20AGRON000203.pdf?sequence $=1 \&$ isAllowed $=$ y

Somarriba, E; Astorga, C; Vásquez, N; Cerda, R; Orozco, L; Quesada, F. (2010). Injertos y otras técnicas de propagación del cacao (Primera ed.). Turrialba-Costa Rica: CATIE.

Vera, C., López, J. (2007). Propagación vegetativa por injerto de concordia (Grevillea robusta Cunn). XXII
Jornadas Forestales entre Ríos. INTA EEA Bella Vista-Argentina. 5p.

Vera, J., Moran, L. (2012). Influencia de la edad del patrón en el cacao (Theobroma cacao L.) sobre el prendimiento de los injertos EET575, EET- 576 y EET- 103. Tesis Ing. Agrícola. Escuela Superior Politécnica Agropecuaria de Manabi Calceta. Ecuador. 50-77p. 